\section{Culture and assessment of manic symptoms*}

\author{
PAUL MACKIN, STEVEN D. TARGUM, AMIR KALALI, DROR ROM \\ and ALLAN H. YOUNG
}

\begin{abstract}
Summary Cultural background may influence the perception of psychiatric symptoms. We examined the effects of cultural biases on the identification of manic symptoms using the Young Mania Rating Scale. Two video interviews, each with an American person with mania, were shown to psychiatrists from three countries (US, UK and India). Total scores on the scale differed significantly between the US and UK $(P<0.00 I)$ and between India and UK $(P<0.001)$ rater groups. Overall, differences between India and US rater groups were less marked ( $P=0.28$ ). These differences suggest that cultural biases influence the interpretation of manic symptoms.
\end{abstract}

Declaration of interest S.T. and D.R. are employees of PharmaStar.

It has previously been noted that 'behaviour pertinent to psychiatry is relative to culture, and cultural differences must enter to some extent into the definitions and perceptions of psychiatric disorders' (Savage et al, 1965). The Amish Study of affective disorders (Egeland et al, 1983) investigated the impact of the masking or modification of psychiatric symptoms by social definition and cultural overlay. It was reported that the correct diagnosis was hindered by the interpretation of symptoms of grandiosity, excessive activity, form of thought, thought content and paranoid features. Other studies have also reported that cultural background affects the presentation and diagnosis of bipolar disorder

*Presented at the 157th Annual Meeting of the American Psychiatric Association, New York, 5 May 2004.
(Strakowski et al, 1996; Kirov \& Murray, 1999).

Psychiatric rating instruments are often used in clinical and research settings to quantify objectively the presence and severity of the specific, individual symptoms and behavioural aspects of the disorder being evaluated. The psychometric properties of these rating instruments are typically established in circumscribed patient populations drawn from the geographical locality of the scale developers. The adaptation of the scale to related psychiatric disorders or to populations from other cultures, using different languages and contexts, may affect the validity of the instrument. We used the Young Mania Rating Scale (YMRS; Young et al, 1978) to explore inter-cultural biases between English-speaking raters from three countries (UK, USA and India) when evaluating acute mania in two American patients.

\section{METHOD}

Two videotaped interviews, each with an American with mania and clearly in an abnormal mental state, were shown to a total of 126 trained English-speaking clinician raters from three different countries: 20 from the UK, 24 from India and 82 from the US, as part of a rater qualification programme for conducting global clinical trials. Although there were training and educational differences as well as the obvious geographical differences, all raters had previous clinical experience with bipolar disorder and the YMRS. Raters were asked to observe and independently score each of the YMRS items for both patients. Raters were told that their scores would be compared with those of other raters for interrater reliability. All participants gave their fully informed consent to participate in this study, following explanation of the protocol.
The YMRS includes 11 items rated with increasing severity from 0 (absent symptoms) to 4 or 8 , depending on the item. The first YMRS video interview examined a woman with bipolar disorder (patient A) who was over-enthusiastic about a new job in a department store, advising women on fashion accessories. The second interview examined a man (patient B) who talked quickly, expressed grandiose ideas, had difficulty sitting still through the interview, was socially inappropriate at times and seemed unconcerned about his behaviour.

Analysis of variance (ANOVA) models were used in the statistical analysis. For each individual patient, each item on the YMRS, as well as the mean total score, was analysed separately in a one-way ANOVA with country (US, UK or India) as the factor in the model. For the overall analysis, a two-way ANOVA with country and patient as factors was used. F-test scores were first reported to test for differences between the three countries, and pairwise contrasts (of each vignette across different countries) were used to test for differences between individual pairs of countries. Results are presented as mean (s.e.). Statistical significance is defined as $P<0.05$.

\section{RESULTS}

Figure 1 shows the distribution of mean total YMRS scores for each country for both participants. For patient A, mean total scores were: India 30.5 (0.78); UK 20.6 (0.58); US $31.6(0.33)$. Total scores for patient B were: India 40.8 (0.68); UK 27.1 (0.97); US 38.6 (0.32). Total YMRS scores differed significantly on both interviews between the US and UK raters $(P<0.001)$, India and the UK $(P<0.001)$, and on one interview between the US and Indian raters groups $(P=0.004)$. Overall analysis revealed that there was a significant difference in mean total YMRS scores across countries $(P<0.001)$, and comparison of individual countries revealed differences between India $v$. UK $(P<0.001)$ and $\mathrm{UK} v$. US $(P<0.001)$, but not India $v$. US $(P=0.28)$.

Individual item analysis revealed significant differences between countries on 10 of the 11 YMRS items on each of the two interviews, the direction of these differences reflecting overall mean total score for each individual country. The most profound differences were noted for mood elevation 
(item 1; $P<0.001$ ), irritability (item 5; $P<0.001$ ), thought content (item 8; $P<0.001$ ), and disruptive-aggressive behaviour (item 9; $P<0.001$ ).

\section{DISCUSSION}

This YMRS was developed with the participation of people with mania from New York State. Hitherto the impact of differing cultures on the assessment of manic symptoms using this rating instrument has not been quantified. Our data reveal that English-speaking psychiatrists from three different countries showed a marked disparity in detecting and rating manic symptoms, reflecting the distinctive clinical perspectives of the raters from each country. Indian raters saw the manic behaviour of the American patients as significantly more ill and inappropriate than did American raters. Conversely, the British raters generally rated these same American patients with mania significantly lower compared with the American raters.

Although only two cases were analysed, the differences in scores between the three countries suggest that intercultural biases affect the interpretation of manic symptoms. We cannot exclude the possibility that other factors, in addition to cultural background, may also have influenced these results. Age, gender, psychiatric training, years of experience, etc., may have
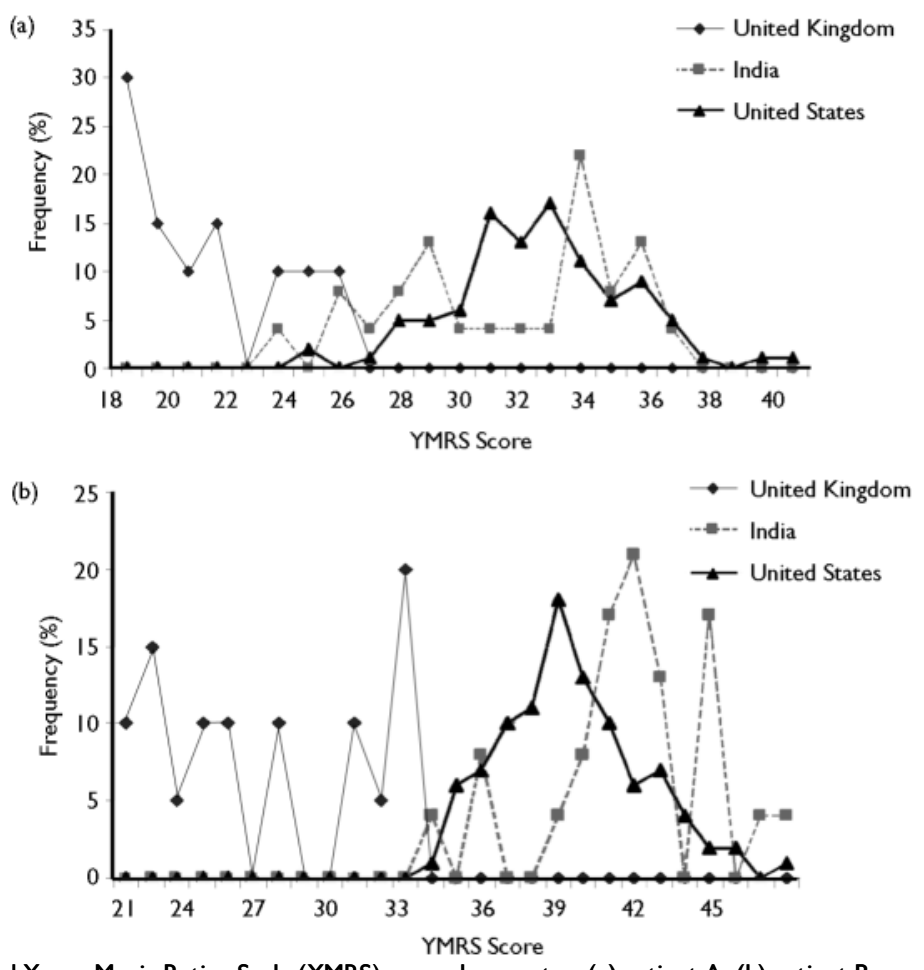

Fig. I Total Young Mania Rating Scale (YMRS) scores by country: (a) patient A; (b) patient B.

PAUL MACKIN, MB BS, PhD, MRCPsych, Department of Psychiatry, University of Newcastle upon Tyne, UK; STEVEN D. TARGUM, MD, PharmaStar, Wayne, Pennsylvania, USA; AMIR KALALI, MD, University of California, Irvine, USA; DROR ROM, PhD, PharmaStar, Wayne, Pennsylvania, USA; ALLAN H. YOUNG, MB ChB, MPhil, PhD, FRCPsych, Department of Psychiatry, University of Newcastle uponTyne, UK

Correspondence: Dr Paul Mackin, School of Neurology, Neurobiology and Psychiatry, University of Newcastle uponTyne, Royal Victoria Infirmary, Queen Victoria Road, Newcastle uponTyne NEI 4LP, UK. Tel: +44 (0)।9I 282 4382; fax: +44 (0)।9I 282 0485; email: paul.mackin@ncl.ac.uk

(First received 8 June 2005, final revision 27 October 2005, accepted 2 December 2005)

acted as confounding variables, in addition to other common evaluation errors (e.g. the halo effect or logistical errors). However, unless all these factors are controlled for, similar variability is likely to be present when rating patients in routine clinical practice or in research studies.

Although preliminary, these data have potentially important implications not only for diagnostic and epidemiological studies, but also for the design of clinical drug trials in which rating instruments are used to assess baseline symptom severity and improvement. Large epidemiological studies such as ÆSOP (Aetiology of Schizophrenia and Other Psychoses) have reported the incidence of bipolar disorder in three UK cities (Lloyd et al, 2005). In the ÆSOP study, cases were defined by a group of clinicians from multicultural backgrounds, and an assumption was made that manic symptoms are universal phenomena and equally detectable. Our data suggest that the cultural background of the clinician

\section{- United Kingdom}

- - 들-- India$$
\text { . }
$$

(1)

may have a direct influence on the diagnosis of bipolar disorder.

With regard to multicentre clinical drug trials, these data suggest that potential participants in studies of people with mania are more likely to be enrolled if assessed by a psychiatrist from India or the US rather than a psychiatrist from the UK, if a YMRS minimum score is stipulated as an entry criterion. Conversely, participants rated by a psychiatrist from India or the US are less likely to satisfy conventional criteria for improvement (i.e. an improvement of $50 \%$ or more on YMRS score) than those rated by a psychiatrist from the UK. Trial designers recruiting sites and raters from multiple countries need to consider the potential confounding impact of cultural bias when evaluating data generated from such studies.

These findings should prompt further discussion, and other large studies using patients in real-life clinical settings and clinicians from other cultural backgrounds are needed.

\section{REFERENCES}

Egeland, J. A., Hostetter, A. M. \& Eshleman, S. K. (1983) Amish Study, III: the impact of cultural factors on diagnosis of bipolar illness. American journal of Psychiatry, 140, 67-7I.

Kirov, G. \& Murray, R. M. (1999) Ethnic differences in the presentation of bipolar affective disorder. European Psychiatry, 14, 199-204.

Lloyd, T., Kennedy, N., Fearon, P., et al (2005) Incidence of bipolar disorder in three UK cities. Results from the AESOP study. British Journal of Psychiatry, 186, $|26-13|$.

Savage, C., Leighton, A. \& Leighton, D. (1965) The problem of cross-cultural identification of psychiatric disorders. In Approaches to Cross-Cultural Psychiatry (eds J. Murphy \& A. Leighton), p. 4. New York: Cornell University Press.

Strakowski, S. M., McElroy, S. L., Keck, P. E., et al (1996) Racial influence on diagnosis in psychotic mania. Journal of Affective Disorders, 8, 157-162.

Young, R. C., Biggs, J.T., Ziegler, V. E., et al (1978) A rating scale for mania: reliability, validity and sensitivity. British Journal of Psychiatry, I33, 429-435. 